\title{
The brain-gut axis in gastrointestinal cancers
}

\author{
Orna Alpert $^{1,2}$, Leonid Begun ${ }^{1}$, Tony Issac ${ }^{1,2}$, Ramon Solhkhah $^{1,2}$ \\ ${ }^{1}$ Department of Psychiatry, Jersey Shore University Medical Center, Neptune, NJ, USA; ${ }^{2}$ Department of Psychiatry \& Behavioral Health, \\ Hackensack Meridian School of Medicine at Seton Hall University, Nutley, NJ, USA \\ Contributions: (I) Conception and design: O Alpert, T Issac; (II) Administrative support: O Alpert, T Issac; (III) Provision of study material or \\ patients: O Alpert, T Issac; (IV) Collection and assembly of data: O Alpert, T Issac; (V) Data analysis and interpretation: O Alpert, T Issac; (VI) \\ Manuscript writing: All authors; (VII) Final approval of manuscript: All authors. \\ Correspondence to: Dr. Orna Alpert. Department of Psychiatry, Jersey Shore University Medical Center, Neptune, NJ, USA.
}

Email: orna.alpert@hackensackmeridian.org.

\begin{abstract}
Recent developments have given more credence into the brain-gut-microbiota axis and its role in the development of tumor genesis. The microbiota have multiple functions including maintenance of the epithelial barrier, immune response, digestion, cortisol regulation, and control of neurotransmitters and their metabolism [e.g., serotonin, dopamine, noradrenaline and gamma-Aminobutyric Acid (GABA)]. Changes in gut microbiota can interfere with homeostasis leading to dysbiosis microbiota, which is linked to colorectal cancer. Microbiota composition can cause pronounced effect on medical interventions including medications, chemotherapy, and radiation. Altered primary immune system is associated with microbiota disassociation and development of colorectal cancer. This article reviews the current research in brain-gut axis with focus on microbiota and its role in the development of gastrointestinal cancers. We conducted a literature review on PubMed, Cochrane, and Science direct using English language. We begin by reviewing the brain-gut axis and its function and then discuss its effect on the development of gastrointestinal cancers. We reviewed 70 manuscripts and found association between microbiota dysfunction and development of colorectal cancers predisposing to psychiatric manifestations. Lasting disturbances in the microbiota can lead to systemic inflammation with implications on disease development or treatment modifications. These disruptions of the intestinal flora can play an important role in the pathogenesis of cancers. Most psychological reactions to cancer are similar across cancer types but each cancer when examined individually has its own unique features associated with it. Correlation between fear of recurrence and the level of pathological distress is viewed as an indicator of overall adjustment to cancer survival.
\end{abstract}

Keywords: Depression; anxiety; PTSD; Gastrointestinal cancer; colorectal cancer; chemotherapy; tumorgenesis; microbiota; microbes; brain-gut axis; systemic inflammation

Submitted Jan 11, 2020. Accepted for publication: Jun 20, 2020.

doi: 10.21037/jgo-2019-gi-04

View this article at: http://dx.doi.org/10.21037/jgo-2019-gi-04

\section{Introduction}

The term brain-gut axis was initially defined in the literature as early as the 1960s, which lead to the concept of ongoing communication between the gut and the brain. The autonomic regulation within the gut is facilitated by the parasympathetic, sympathetic, and enteric nervous systems, and neuroendocrine factors (1). The communication between the brain and the gut is bidirectional with the gut synthesizing and secreting multiple neuroactive substances that are able to cross the blood-brain barrier and affect the brain. In-turn, neuroactive molecules originated in the brain can exert effects on the gut via the sympathetic and parasympathetic systems or the humoral pathway (2). These neuropeptides include substance $\mathrm{P}$, calcitonin gene related peptide, and neuropeptide Y (3).

This indicates that the nervous system and the humeral pathway are able to send signals from tumor cells to the 
brain, which in turn is followed by signals from the brain modulating the tumor growth factor in the gut (3). Recent studies have also shown that neural signaling substances are involved in tumorigenesis and progression of GI malignancies (4).

The brain-gut axis includes the following components: the central nervous system (CNS), the autonomic nervous system (ANS), which consists of the sympathetic and parasympathetic systems, and the gut microbiota (1). The CNS is the first component of the brain-gut axis. It was demonstrated that stimulation of certain areas of the CNS could promote proliferation and metastasis of cancer cells. For example, electrical stimulation from the lateral hypothalamus can enhance the cytotoxicity of natural killer cells (1), and an injury to the lateral hypothalamus can suppress cytotoxicity. The opposite effect is observed with electrical stimulation of the ventromedial hypothalamus. Hypothalamus-pituitary-adrenal axis activation in depressed patient can induce deficiency in DNA repair that can stimulate tumorigenesis by activation of neuropeptide and neurotransmitter metabolism (1). We often see that when the brain-gut axis is activated by tumor cells, the brain responds to the cancer cells with neuroendocrineimmune and behavioral reactions, involving neuropeptides, neurotransmitter metabolism, regional brain stimulation and behavioral changes (5).

The ANS is the second component of the braingut axis. Its role in the development of GI cancers is complex. The activation of sympathoadrenal axis promotes tumor genesis in the GI system and sympathectomy with 6-hydroxydopamin can reduce the incidence in colon cancer in rats (6). The inactivation of the parasympathetic system by vagotomy could increase the risk of gastric cancer (6). Studies have shown that vagotomy, or inactivation of the vagus nerve by upregulating the level of substance $\mathrm{P}$, can promote liver, kidney, and lung metastasis. Therefore, an intact vagus nerve may protect against metastasis. In addition, other factors such as Helicobacter pylori, hypochloridria, smoking and bile reflux, can contribute to gastric cancer (7) (Figure 1).

Research has shown that many GI tumors including colorectal cancer, gastric cancer, and biliary cancer can be infiltrated and innervated by nerves (9). This perineural tumor invasion is important because it has a prognostic value. Worse median overall survival is observed in gastric cancer tissues that are capable of perineural invasion as it relates to vascular invasion and lymph nodes metastasis (10). Nerve cells involved in perineural invasion can secrete neurotransmitters or neuropeptides, which can promote tumor proliferation (10).

Neuropeptide and neurotransmitters have a role in the modulation of tumor proliferation, migration, invasion, and angiogenesis (11). Those include epinephrine/ norepinephrine, acetylcholine, serotonin (12), GABA (13), and BDNF. Recent studies have also shown that neural signaling substances are involved in tumorigenesis and progression of GI malignancies (14).

The third component of the brain-gut axis is the microbiota, which is the commensal bacteria of the GI system that populate the gastrointestinal and colonic epithelia (15). Gut microbiota consists predominantly of the Bacteroidetes and Firmicutes phyla bacteria (90\%) with the remaining $10 \%$ consisting of organisms such as fungi, archaea, viruses, and bacteria such as Actinobacteria, Proteobacteria and Verruca microbiota phyla (16). The microbiota play a role in multiple functions in the gut including the maintenance of the epithelial barrier, the gut metabolism such as digestion, vitamin synthesis, and immunology (16). They are involved in cortisol regulation, and control of serotonin, dopamine, noradrenaline, and GABA metabolism (16).

The microbiota is unique for every individual, and the diversity in microbiota is influenced by age, diet, environmental factors, such as disease affecting the individual, and the usage of antibiotics and other medications. The colonization of the newborn digestive system occurs during delivery when the infants are exposed to maternal microbiota (17). Lactobillus is dominant after vaginal delivery (18) and Staphylococcus and Priopionibacterium are more prevalent in neonates born via cesarean section (19).

The brain exerts control on the gut via autonomic and neuroendocrine pathways. An abnormal interaction between the two can result in gastrointestinal diseases, such as Inflammatory Bowel Syndrome that is associated with pain anxiety and depression. The brain and the gut axis employs four major communication systems, including, neuro-messages carried by the vagal and spinal afferent neurons, immune messages carried by cytokines, endocrine messages carried by gut hormones for microbial factors that can reach the brain directly via blood stream or indirectly by other transmission pathways (15). The communication systems mentioned above are present in various locations throughout the gastrointestinal tract. Those have important and vital functions including the ability of the gut to distinguish between toxins and to maintain 


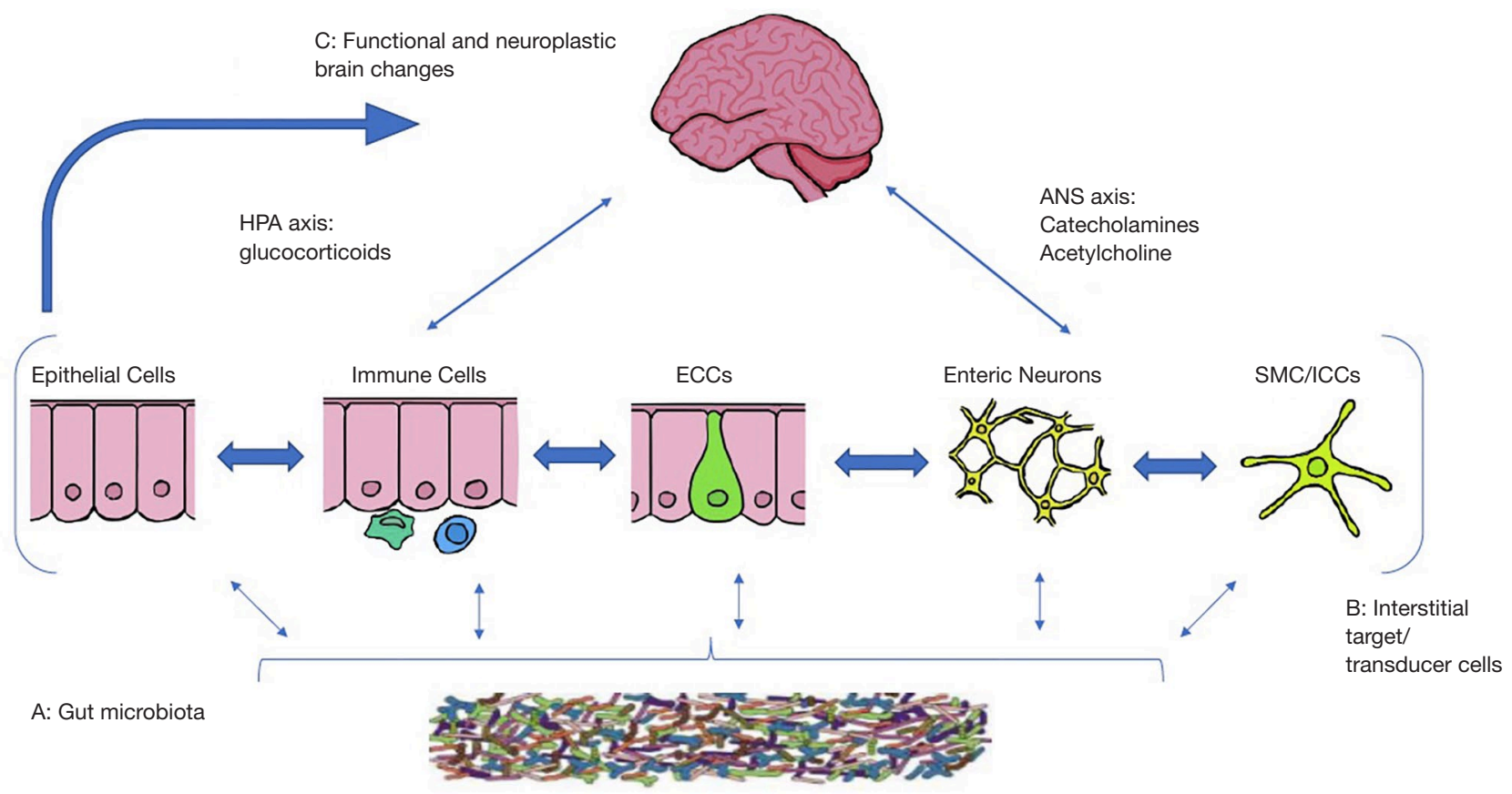

Figure 1 Key components of brain gut microbiota axis. A network of specialized target/transducer cells in the gut wall functions as an interface between the organism and the gut lumen. In response to external and bodily demands, the brain modulates individual cells (ECC, enterochromaffin cells; SMC, smooth muscle cells; ICC, interstitial cells of Cajal) within this network via the branches of the autonomic nervous system (ANS) (sympathetic and parasympathetic/vagal efferents) and the hypothalamic pituitary adrenal (HPA) axis. Such modulation can be transient (e.g., in response to transient perturbations) or longlasting (in response to chronically altered brain output). The microbiota is in constant bidirectional communication with this interface via multiple signaling pathways, and this communication is modulated in response to perturbations of the microbiota, or the brain. The integrated output of the brain gut microbial interface is transmitted to the brain via multiple afferent signaling pathways, including endocrine and neurocrine (vagal, spinal afferents) pathways. While acute alterations in this interoceptive feedback result in transient functional brain changes, chronic alterations are associated with neuroplastic brain changes." (8).

homeostasis, especially through the immune system. Those communication pathways between the nervous system and the gastrointestinal tract involve neuropeptide and similar molecule (2). These peptides exhibit modulatory effects on the gastrointestinal tract endocrine cells which can produce upwards of 20 different hormones. These hormones can have an effect on the microbiota which is located in very close proximity within the gastrointestinal mucosa. The gut hormones in addition to immune mediators play a role in communication between the brain and the microbiota. Evidence of this communication can be seen when we look at metabolites circulating in the blood which are derived from the intestinal microbiota (20). It is also important to recognize that the presence or absence of the microbiota, can affect the variability of metabolites such as peptides present in the brain.
Studies have shown that there are three phases in the microbiota gut colonization in the infant that begin at three months of age and continues for about four years. The first phase, developmental phase, is from 3-14 months. The second phase, a transitional phase, is from 15-30 months, and the third phase, stable phase, from 31 months onward. Maturation of microbiota continues until age 20 (19). During the first months of life, the microbiota is of reduced diversity. Infants that were breast-fed had higher concentration of Bifidobactrerium, which has probiotic properties (19). As the infant is exposed to variety of foods, the microbiota becomes more diverse and a higher concentration of Firmicutes Phyla, typically found in adults, is now populating in the infant's gut. Babies born vaginally are also exposed to a temporary increase in concentration of Bacteroides bacteria that increases the gut diversity and 
maturation during the first year of life (19).

Diet can influence the arrangement of the intestinal microbiome. Poor prenatal diet (high fat, low fiber) can affect the diversity and composition of microbiota, which can increase the incidence of allergic sensitization (allergic rhinitis, eosinophilia, asthma) and metabolic diseases in children (21). There is evidence that dietary supplementation of perinatal prebiotic or probiotic supplements could modify the composition of gut microbiota in mothers and infants (22). Children fed diet rich in fibers tend to have a healthy gut microbiota, and a diet rich in fat in the first years of life can decrease the total amount of Bacteriodetes (11). The environment of the microbiota is sensitive to changes in $\mathrm{pH}$, oxygen, nutrients, and integrity of mucous membrane (23). Gram negative like Bacteroides and Gram positive like firmicutes are the main microbiota in healthy adults (18). Other organisms like Proteobacteria and Fusobacteria are less common (24).

Any change in the gut microbiota can interfere with homeostasis leading to a state of dysbiosis microbiota, which is linked to colorectal cancer via various mechanisms (25). Dysbiosis microbiota include bacterial species such as Bacteroids fragilis, Streptococcus bovis, Helicobacter pylori, Enterococcus faecalis, Escherichia coli, Clostridium septicum and Fusobacterium spp have procarcinogenic properties (25). The microbiota has an effect on metabolism of chemotherapy, and immunotherapy (15).

In the recent years, an interest in "pharmacomicrobiomics", the effect of microbiota on drug activity and metabolism, has grown, and a large number of studies have focused on the role of microbiota on various drug metabolism (26). It is believed that the microbiota exert its effect on drug metabolism through enzymatic effect on the drug or by direct effect on host gene expression of enzymes involved in the drug metabolism.

Of particular relevance in Oncology, studies have shown a close relationship between microbiota, chemotherapy, and immunotherapy agents. Through immune modulation, chemotherapeutic agents can affect the growth of gut microbiota (27). It also became apparent that irradiation could produce oxygen radicals leading to cytotoxic effects on the epithelium with ulcerative lesions and microbial cell damage, which can alter and disrupt the microbiota population. The altered microbiota can worsen radiotherapy-induced mucositis with an increased risk for infection and other clinical complications (28).

The gut microbiota has an effect on the metabolism of chemotherapeutic and anticancer drug response such as 5-Flurouracil, oxaliplatin, and irinotecan. Microbiota can influence drug absorption, breakdown, and toxicity (29). It can both promote tumor suppression though synergistic effect and can augment tumor genesis by interfering with treatment and drug efficacy (30). Antibiotics can alter gut microbiota, which can reduce drug efficacy of cyclophosphamide and platinum based compounds like oxaliplatin and cisplatin (31). Microbiota can also exert an effect on metabolism of medications such as digoxin, methotrexate, diabetic medications, risperidone, levodopa, metronidazole, omeprazole, nitroglycerine, and clonazepam (27).

Research has shown that chemotherapy can increase the growth of Bacteroids species, Escherichia and Enterococcus Faecium and decrease the growth of few Clostridium species, Bifidobacterium, Lactobacillus, and others (32). When the cytotoxic effect of chemotherapy induce dysbiosis microbiota, this dysbiosis can spread microbiota into the blood stream leading to increased morbidity and mortality rates in colorectal cancer patients (33). A commensal bacterium, a type of microbiota, interacts with TLR and nuclear factor kappa B, promoting a protective effect on the colorectal epithelial cells by enhancing innate immune response (33).

The activation of those mechanisms is essential in protecting the intestinal mucosa barrier against various injuries (33). Studies have shown that the use of probiotics can deter the inflammatory effects and mucositis caused by chemotherapy, and Lactobacillus probiotic can help to reduce mucosal injury (34). Another study showed that supplementation with natural flora can mitigate the effect of chemotherapy as well. Additionally probiotic supplementation to patients receiving 5-FU, who developed severe diarrhea, showed favorable outcome in reduction of diarrhea and electrolytes imbalance (35). In summary, probiotics can lower chemotherapy induced intestinal mucositis; therefore, supplementation with probiotics should strongly be considered (15).

Despite the multiple diverse treatment options at our disposal today, the mortality rates for colorectal cancer remain elevated. There is an urgency to identify risk factors and biomarkers to improve treatment outcomes overall. Recent research focuses on the gut microbiota and the role of development of colon cancer (36). Other key factors that influence colon cancers are host genetics (37), diet (38), and inflammatory dysregulation (39).

Tumor genesis of colorectal cancer is associated with mutations in suppressor genes and onco-genes leading to the differentiation of normal cells to malignant cells. The 
development of colon cancer is commonly initiated by mutations in Adenopatous Polyposis Coli (APC) tumor suppressor gene (40). The APC protein is a negative regulator of WNT/B-Catenin. APC mutations contribute to the dysregulation of WNT/B-Catenin initiating alterations in microbiota that can result in development of polyps and tumorigenesis (41).

The brain-gut axis and its components have effects on gastrointestinal cancers, those include: esophageal cancer, gastric cancer, colorectal cancer, pancreatic, and liver cancers. In this paper we will focus on colorectal cancers due to its most important and extensive representation in the brain-gut axis.

Research has shown that microbiota can modulate human health and pathology including cancer proliferation. The microbiota can effect cancer growth initiation and proliferation through various mechanisms. Microbes can produce free radicals that lead to DNA damage, an example of this is, B fragilis that produce reactive oxygen that can cause colon cancer by damaging the host DNA (42). Microbiota can also increase cell proliferation, which was observed by the fact that H. Pylori can increase cell proliferation and induce tumor genesis. As most of the world's population is colonized with $H$. Pylori, however only small percentage of people develop GI cancers and this may be due to the carcinogenic phenotypes of the host which allow the $H$. pylori to initiate and proliferate cancer cells in the stomach (11).

\section{Colorectal cancer}

According to the American Cancer Society, colorectal cancer is the third most common cancer in both sexes. There are 101,420 new cases of colon cancer and 44,180 new cases of rectal cancer expected in the US in 2019. It is the second leading cause of cancer death worldwide with an estimate of about 1 million people being diagnosed every year (43). The highest incidence of colorectal cancer is found in central and northern Europe, Australia, New Zealand, Northern America, and Eastern Asia. Colorectal cancer is common in the developed countries and 50-65\% of new cases occur in Europe and the Americas.

Due to improvement in early detection, diagnosis, and treatment, survival of patients with colorectal cancer has improved, and $50 \%$ of patients will survive at least 5 years (43). About 11 million survivors are living in the US and that population is growing (44). Patients survival is largely depended on treatment which include surgery, followed by chemotherapy and/or radiation therapy (43). The use of 5 -Fluorouracil has resulted in an improvement in survival of approximately $30 \%$ with additional $20 \%$ improvement in survival when oxaliplatin was added to patients with stage III colon cancer. Over $90 \%$ of invasive colorectal cancers are diagnosed in patients over 50 years old with $67 \%$ of cases diagnosed in patients 65 years and older (44).

Most psychological reactions to cancer are similar across cancer types but each cancer when examined individually has its own unique features. Particular issues that are characteristic for colorectal cancer are: stoma management, body image, bowel symptoms, low energy, and financial concerns (45). Colon cancer recurrence is common, and $90 \%$ of cases will show recurrence in the first 2 years post treatment (43). The fear of recurrence contributes to the difficulties faced by survivors, even after initial remission has been achieved. The anxiety is acute in the first few months after completing treatment, and it may continue for few years $(44,46)$. Studies have shown correlation between fear of recurrence and the level of psychological distress, which was viewed as indicator of overall adjustment to cancer and survival (47).

As more patients survive, physical and mental health concerns due to cancer treatment emerges (including PTSD, anxiety, and depression) leading to new challenges that can affect the patient's quality of life (QOL). We define QOL as a "subjective evaluation of one's personal satisfaction with overall health and well-being (48)." QOL is measured by physical, psychological, and social functioning. When patients talk about QOL, the most prevalent concerns they express are recurrence of the cancer (68\%), followed by fatigue (67\%), and sleep disturbance (48\%) (44).

Cancer survivors often report poorer health ratings. Female long-term cancer survivors endorse difficulty in physical activities such as walking; climbing stairs, and maintaining housekeeping compared to same age females without cancer. Male cancer survivors reported more physical limitations in activities such as sports, shopping, and social events compared to same age adults without cancer (43). For elderly long-term survivors, anxiety and depression are often a significant complaint, and they have worse physiological outcomes and have increased use of mental health services compare to elderly without cancer (43).

\section{Post-traumatic stress disorder (PTSD)}

Patients who are being diagnosed with cancer, being treated for the cancer, and surviving it often reported 
feeling stressed and overwhelmed (49). This experience can lead to symptoms consistent with PTSD that include intrusive thoughts, nightmares, re-experiencing the trauma (flashbacks), hyper-arousability, avoidance behavior, and change in mood and cognition. The incidence of PTSD is variable among cancer patients, and it ranges between $0 \%$ to $32 \%(49)$. Anxiety and depression are comorbid with PTSD (50). Following a diagnosis of cancer, the prevalence of anxiety tends to be high. In one study, 19\% and $22.6 \%$ of patients showed clinical and subclinical anxiety at diagnosis (51). It is unclear whether a traumatic experience of cancer can increase the risk of PTSD or a previous history of PTSD can increase the risk of cancer. It has been evident clinically that cancer can be experienced as traumatic event leading to PTSD in minority of patients (52).

It became apparent recently, that many cancer survivors do not report an increase in stress and poor adjustment, but report positive outcomes and periods of psychological growth following a cancer diagnosis and treatment (49). Tedeschi and Calhoun described a term Posttraumatic Growth (PTG) as a positive life change after experiencing stressful and traumatic events including cancer (53). The positive changes are described as personal growth, increase in strength, and an appreciation of life and spirituality (54). The term PTG was seen in cancer cases, but it also in survivors of HIV infection and sexual assault (54). Indices of cancer related distress, such as perceived stress and perceived life threat, are predictive of high level of PTG, but indices of general distress or well-being were not correlated with PTG. It is unclear if there is a correlation between psychological adjustment and PTG after cancer, but as with any other traumatic events, both can promote meaningful growth (55). In other words, undergoing cancer diagnosis, treatment has a potential for both positive and negative outcomes. Tedeschi and Calhoun wrote that distress and growth can coexist and initial elevated level of stress can stimulate subsequent growth (49).

They also hypothesized that since PTSD and PTG have similar pathways, both occur in response to an initial traumatic event that lead to significant psychological distress. These psychological distresses consist of unwanted intrusive thoughts of the trauma, which appear as rumination but are an attempt to process the traumatic event. According to multiple sources in the literature, a certain amount of distress is essential to stimulate growth and rumination as a central cognitive process to promote PTG (55).

This process of rumination is a cognitive processing with positive and negative outcomes. The positive processing facilitates adaptation to trauma, which increases growth and meaningful outcomes while negative processing facilitates distress and depression. Intrusive and disruptive thoughts that are uncontrolled and indicative of stress response to trauma are negative processing but thoughts that are brief and controlled can be positive and beneficial. When processing the trauma is deliberate and effortful, it result in decrease in distress and increase in PTG (49). Therefore, depending on the cancer survivors cognitive processing, the outcome can result in positive growth and development versus the development of a PTSD picture.

\section{Integration of body image}

Surgical intervention with resection of the affected part of the colon or rectum is often the first line of treatment of colorectal cancer. Following a partial or total colectomy, the creation of a stoma, which is an opening at the skin to remove fecal material, is often required. Colostomies can be temporary and reversible or permanent depending on the extent and the nature of the disease. Over the years, there has been much interest in the psychological effects of having a permanent stoma on patients with colorectal cancer (56). Studies have shown significant difference in depression, social functioning, body image and sexual functioning between patients with stoma and patients without a stoma. Likewise patients who underwent bowl resection with stoma placement were likely to develop body image disturbance which subsequently lead to depression (57).

Body image dissatisfaction is common in other disease such as breast cancer or rheumatological disease, and it was a strong predictor of depressive symptoms, anxiety, and distress. Sharpe et al. recruited 99 patients with colorectal cancer: 34 patients had stoma placement and $65 \operatorname{did}$ not (56). The study attempted to determine the effect of having a stoma for the treatment of colorectal cancer on body image and whether body image disturbance can predict the development of subsequent psychological distress. Their work showed that there is correlation between patients who received a stoma and their body image (56). Patients with colorectal resection and stoma placement showed more body image disturbance following the surgery than patients who did not receive a stoma. Furthermore, Sharpe et al. showed that these body image disturbances did not fade away with time but instead continued to progress and worsen over time (56). To further support these findings, Ross et al., found that patients who received a late stoma 
had a significant increase in depressive symptoms (57).

The baseline level of body image disturbance before treatment was a predictor of anxiety and distress once treatment began. Sharpe found similar results in their study as the presence of a stoma was associated with poorer body image and this image did not adjust with time. This evidence highlights the importance of assessing body image issues when diagnosing, treating, and following up with patients with colorectal cancer as these issues can clearly lead to increase in depression and general distress (56).

\section{Surviving cancer treatment}

Rates of cancer treatments continue to improve across the board. As such, there is a great deal of information that we still need to learn and understand about the battle survivors face once they are in remission. There is an effect known as "guarding" which was suggested and investigated by Taylor et al. (43). They identified guarding as "when a survivor becomes vigilant to their bodies changing state, as professional support declines and time post treatment extends." These findings can manifest as uncertainty over one's future, loss of confidence in one's health, fear of recurrent disease, perceived loss over body control, and the need for outside and professional reassurance. There are three main forms in which guarding can present itself: body monitoring, risk management, and seeking reassurance (43). Body monitoring is initially a required part of one's treatment as the body responds and adjusts to surgery, chemotherapy, and radiotherapy treatments; however, as time post treatment progresses, the patients tend to selfmonitor their bodies for nuances of their recovery (41). This can have significant effects on daily life, food intake, and social interactions. Simple symptoms like headaches can be interpreted as an ominous finding. Patients can experience a loss of autonomy and become fully dependent on healthcare providers for interpreting their body function that can lead to frequent healthcare utilization and increased cost (41).

The next coping mechanism is managing risk. This can present as an appropriate post treatment behavior as patients follow-up with their healthcare providers recommendations in an attempt to make life style modifications. However, it can lead to fearful and overly cautious behavior that is incongruent with the patient's actual abilities after remission (41). Many patients may tend to avoid many activities due to fear of disease recurrence. This can manifest as unreasonable diet modifications, being overly sensitive to medical recommendations on the news, or even doubting the advice of their medical team (41). Patients in these circumstances often believe that they can help prevent recurrence by avoiding or managing their activities.

Finally, seeking reassurance is another double-edged sword that can present itself during the guarding phase (41). During initial treatment, patients often benefit from constant reassurance and support from their treatment team and network, but as time progresses, patients can find sharing their experience can be anxiety-inducing as many individuals volunteer suggestions and advice (41). A fear of this can cause patients to avoid sharing their experiences and worries, leading to internalization of their discomfort until symptoms of anxiety and depression emerge (41).

\section{Conclusions}

Mounting evidence exists in support of the brain-gut axis and its involvement in tumor genesis, proliferation and growth. Further research is needed in order to comprehend the intricate balance between the three main components of the brain-gut axis: the CNS, the ANS, and the gut microbiota. In this paper we focused on the numerous effects on microbiota leading to a state of dysbiosis that can predispose to colorectal cancer via various mechanisms. These changes can have effects on metabolism of chemotherapy and immunotherapy. There is also need to elucidate the tumor genesis process of colorectal cancer, as it clearly plays a role with mutations in suppressor genes and onco-genes leading to the differentiation of normal cells to malignant cells. There is an urgency to identify risk factors and biomarkers to improve treatment outcomes throughout all cancer pathologies.

The mortality rates of colorectal cancer are projected to decrease in the coming decades with an increasing number of survivors. This goes to underline the importance of continued efforts to participate in a collaboration of care between multiple specialties. This collaboration requires an in depth comprehension of the challenges faced by patients, survivors, and their support network. Focusing on defining QOL quantification is a viable option moving forward. Much more research is required to further understand the causes that many survivors report as an increase in stress and poor adjustment after positive outcomes, which can stunt their ability for PTG. Development of positive coping mechanism using a biopsychosocial approach can allow better coping with the issues faced by many colorectal cancer survivors living with a stoma, either permanently or temporarily. Finally, there needs to be further 
understanding of the transition faced by each individual as they transition from active disease state to remission. The goal is to avoid the formation of the three-part process described as Guarding, where the positive behavior that was required during treatment can effectively become the chains that keep the patient tethered to the disease.

\section{Acknowledgments}

Funding: None.

\section{Footnote}

Provenance and Peer Review: This article was commissioned by the Guest Editors (John F. Gibbs and Quyen D. Chu) for the series "Global GI Malignancies" published in fournal of Gastrointestinal Oncology. The article has undergone external peer review.

Conflicts of Interest: All authors have completed the ICMJE uniform disclosure form (available at http://dx.doi. org/10.21037/jgo-2019-gi-04). The series "Global GI Malignancies" was commissioned by the editorial office without any funding or sponsorship. Dr. Solhkhah is a Consultant at Alexza Pharmaceuticals, and reports grants from Galen Ltd. Pharmaceuticals, grants from Otsuka Pharmaceutical, grants from Alkermes PLC, grants from LivaNova PLC, outside the submitted work. The authors have no other conflicts of interest to declare.

Ethical Statement: The authors are accountable for all aspects of the work in ensuring that questions related to the accuracy or integrity of any part of the work are appropriately investigated and resolved.

Open Access Statement: This is an Open Access article distributed in accordance with the Creative Commons Attribution-NonCommercial-NoDerivs 4.0 International License (CC BY-NC-ND 4.0), which permits the noncommercial replication and distribution of the article with the strict proviso that no changes or edits are made and the original work is properly cited (including links to both the formal publication through the relevant DOI and the license). See: https://creativecommons.org/licenses/by-nc-nd/4.0/.

\section{References}

1. Di YZ, Han BS, Di JM, et al. Role of the brain-gut axis in gastrointestinal cancer. World J Clin Cases 2019;7:1554-70.

2. Petra AI, Panagiotidou S, Hatziagelaki E, et al. GutMicrobiota-Brain Axis and Its Effect on Neuropsychiatric Disorders With Suspected Immune Dysregulation. Clin Ther 2015;37:984-95.

3. Holzer P, Farzi A. Neuropeptides and the microbiota-gutbrain axis. Adv Exp Med Biol 2014;817:195-219.

4. Ondicova K, Mravec B. Role of nervous system in cancer aetiopathogenesis. Lancet Oncol 2010;11:596-601.

5. Miller AH, Ancoli-Israel S, Bower JE, et al. Neuroendocrine-immune mechanisms of behavioral comorbidities in patients with cancer. J Clin Oncol 2008;26:971-82.

6. Caygill CP, Knowles RL, Hall R. Increased risk of cancer mortality after vagotomy for peptic ulcer: a preliminary analysis. Eur J Cancer Prev 1991;1:35-7.

7. Ekbom A, Lundegardh G, McLaughlin JK, et al. Relation of vagotomy to subsequent risk of lung cancer: population based cohort study. BMJ 1998;316:518-9.

8. Mayer EA, Savidge T, Shulman RJ. Brain-gut microbiome interactions and functional bowel disorders. Gastroenterology 2014;146:1500-12.

9. Amit M, Na'ara S, Gil Z. Mechanisms of cancer dissemination along nerves. Nat Rev Cancer 2016;16:399-408.

10. Duraker N, Şişman S, Can G. The Significance of Perineural Invasion as a Prognostic Factor in Patients with Gastric Carcinoma. Surg Today 2003;33:95-100.

11. Wieczorska K, Stolarek M, Stec R. The Role of the Gut Microbiome in Colorectal Cancer: Where Are We? Where Are We Going? Clin Colorectal Cancer 2020;19:5-12.

12. Tutton PJ, Barkla DH. Influence of inhibitors of serotonin uptake on intestinal epithelium and colorectal carcinomas. Br J Cancer 1982;46:260-5.

13. Bianchi MT, Song L, Zhang H, et al. Two different mechanisms of disinhibition produced by GABAA receptor mutations linked to epilepsy in humans. J Neurosci 2002;22:5321-7.

14. Tsang SW, Auyeung KK, Bian ZX, et al. Pathogenesis, Experimental Models and Contemporary Pharmacotherapy of Irritable Bowel Syndrome: Story About the Brain-Gut Axis. Curr Neuropharmacol 2016;14:842-56.

15. Saeed A, Eshrat FF, Umar S, et al. The Duplex Interaction of Microbiome with Chemoradiation and Immunotherapy: Potential Implications for Colorectal Cancer. Curr Colorectal Cancer Rep 2019;15:98-104. 
16. Evans JM, Morris LS, Marchesi JR. The gut microbiome: the role of a virtual organ in the endocrinology of the host. J Endocrinol 2013;218:R37-47.

17. Schwabe RF, Jobin C. The microbiome and cancer. Nat Rev Cancer 2013;13:800-12.

18. Zou S, Fang L, Lee MH. Dysbiosis of gut microbiota in promoting the development of colorectal cancer. Gastroenterol Rep (Oxf) 2018;6:1-12.

19. Stewart CJ, Ajami NJ, O'Brien JL, et al. Temporal development of the gut microbiome in early childhood from the TEDDY study. Nature 2018;562:583-8.

20. Cenit MC, Sanz Y, Codoner-Franch P. Influence of gut microbiota on neuropsychiatric disorders. World J Gastroenterol 2017;23:5486-98.

21. Dawson SL, Craig JM, Clarke G, et al. Targeting the Infant Gut Microbiota Through a Perinatal Educational Dietary Intervention: Protocol for a Randomized Controlled Trial. JMIR Res Protoc 2019;8:e14771.

22. Shadid R, Haarman M, Knol J, et al. Effects of galactooligosaccharide and long-chain fructooligosaccharide supplementation during pregnancy on maternal and neonatal microbiota and immunity-a randomized, double-blind, placebo-controlled study. Am J Clin Nutr 2007;86:1426-37.

23. Flint HJ, Scott KP, Louis $\mathrm{P}$, et al. The role of the gut microbiota in nutrition and health. Nat Rev Gastroenterol Hepatol 2012;9:577-89.

24. Ajouz H, Mukherji D, Shamseddine A. Secondary bile acids: an underrecognized cause of colon cancer. World J Surg Oncol 2014;12:164.

25. Gagnière J, Raisch J, Veziant J, et al. Gut microbiota imbalance and colorectal cancer. World J Gastroenterol 2016;22:501-18.

26. Haiser HJ, Turnbaugh PJ. Developing a metagenomic view of xenobiotic metabolism. Pharmacol Res 2013;69:21-31.

27. Viaud S, Saccheri F, Mignot G, et al. The intestinal microbiota modulates the anticancer immune effects of cyclophosphamide. Science 2013;342:971-6.

28. Burdak-Rothkamm S, Rothkamm K. Radiation-induced bystander and systemic effects serve as a unifying model system for genotoxic stress responses. Mutat Res 2018;778:13-22.

29. Kuipers EJ, Grady WM, Lieberman D, et al. Colorectal cancer. Nat Rev Dis Primers 2015;1:15065.

30. Ferreira MR, Muls A, Dearnaley DP, et al. Microbiota and radiation-induced bowel toxicity: lessons from inflammatory bowel disease for the radiation oncologist.
Lancet Oncol 2014;15:e139-47.

31. Daillère R, Vétizou $M$, Waldschmitt $\mathrm{N}$, et al. Enterococcus hirae and Barnesiella intestinihominis Facilitate Cyclophosphamide-Induced Therapeutic Immunomodulatory Effects. Immunity 2016;45:931-43.

32. Manichanh C, Varela E, Martínez C, et al. The Gut Microbiota Predispose to the Pathophysiology of Acute Postradiotherapy Diarrhea. Am J Gastroenterol 2008;103:1754-61.

33. Bajic JE, Johnston IN, Howarth GS, et al. From the Bottom-Up: Chemotherapy and Gut-Brain Axis Dysregulation. Front. Behav. Neurosci 2018;12:104.

34. Chang CW, Liu CY, Lee HC, et al. Lactobacillus casei Variety rhamnosus Probiotic Preventively Attenuates 5-Fluorouracil/Oxaliplatin-Induced Intestinal Injury in a Syngeneic Colorectal Cancer Model. Front Microbiol 2018;9:983.

35. Osterlund P, Ruotsalainen T, Korpela R, et al. Lactobacillus supplementation for diarrhoea related to chemotherapy of colorectal cancer: a randomised study. $\mathrm{Br}$ J Cancer 2007;97:1028-34.

36. Chen GY. The Role of the Gut Microbiome in Colorectal Cancer. Clin. Colon Rectal Surg 2018;31:192-8.

37. Belcheva A, Irrazabal T, Robertson SJ, et al. Gut microbial metabolism drives transformation of MSH2-deficient colon epithelial cells. Cell 2014;158:288-99.

38. Structure, function and diversity of the healthy human microbiome. Nature 2012;486:207-14.

39. Zaki MH, Vogel P, Malireddi RK, et al. The NOD-like receptor NLRP12 attenuates colon inflammation and tumorigenesis. Cancer Cell 2011;20:649-60.

40. Rakoff-Nahoum S, Medzhitov R, Rakoff-Nahoum S, et al. Regulation of spontaneous intestinal tumorigenesis through the adaptor protein MyD88. Science 2007;317:124-7.

41. Gur C, Ibrahim Y, Isaacson B, et al. Binding of the Fap2 protein of Fusobacterium nucleatum to human inhibitory receptor TIGIT protects tumors from immune cell attack. Immunity 2015;42:344-55.

42. Whisner CM, Athena Aktipis C. The Role of the Microbiome in Cancer Initiation and Progression: How Microbes and Cancer Cells Utilize Excess Energy and Promote One Another's Growth. Curr Nutr Rep 2019;8:42-51.

43. Taylor C, Richardson A, Cowley S. Surviving cancer treatment: An investigation of the experience of fear about, and monitoring for, recurrence in patients following treatment for colorectal cancer. Eur J Oncol Nurs 
2011;15:243-9.

44. Denlinger CS, Barsevick AM. The challenges of colorectal cancer survivorship. J Natl Compr Canc Netw 2009;7:88393; quiz 94.

45. Baker F, Denniston M, Smith T, et al. Adult cancer survivors: how are they faring? Cancer 2005;104:2565-76.

46. Mullens AB, McCaul KD, Erickson SC, et al. Coping after cancer: risk perceptions, worry, and health behaviors among colorectal cancer survivors. Psychooncology 2004;13:367-76.

47. Ronson A, Body JJ. Psychosocial rehabilitation of cancer patients after curative therapy. Support Care Cancer 2002;10:281-91.

48. Wilson IB, Cleary PD. Linking clinical variables with health-related quality of life. A conceptual model of patient outcomes. JAMA 1995;273:59-65.

49. Salsman JM, Segerstrom SC, Brechting EH, et al. Posttraumatic growth and PTSD symptomatology among colorectal cancer survivors: a 3-month longitudinal examination of cognitive processing. Psychooncology 2009;18:30-41.

50. Palmer SC, Kagee A, Coyne JC, et al. Experience of trauma, distress, and posttraumatic stress disorder among breast cancer patients. Psychosom Med 2004;66:258-64.

Cite this article as: Alpert O, Begun L, Issac T, Solhkhah R. The brain-gut axis in gastrointestinal cancers. J Gastrointest Oncol 2021;12(Suppl 2):S301-S310. doi: 10.21037/jgo-2019-gi-04
51. Linden W, Vodermaier A, Mackenzie R, et al. Anxiety and depression after cancer diagnosis: prevalence rates by cancer type, gender, and age. J Affect Disord 2012;141:343-51.

52. Brewin CR, Andrews B, Valentine JD. Meta-analysis of risk factors for posttraumatic stress disorder in traumaexposed adults. J Consult Clin Psychol 2000;68:748-66.

53. Frazier P, Conlon A, Glaser T. Positive and negative life changes following sexual assault. J Consult Clin Psychol 2001;69:1048-55.

54. Tedeschi RG, Calhoun LG. The Posttraumatic Growth Inventory: measuring the positive legacy of trauma. J Trauma Stress 1996;9:455-71.

55. Tedeschi RG, Calhoun LG. Posttraumatic growth: Conceptual foundations and empirical evidence. Psychol Inq 2004;15:1-18.

56. Sharpe L, Patel D, Clarke S. The relationship between body image disturbance and distress in colorectal cancer patients with and without stomas. J Psychosom Res 2011;70:395-402.

57. Ross L, Abild-Nielsen AG, Thomsen BL, et al. Quality of life of Danish colorectal cancer patients with and without a stoma. Support Care Cancer 2007;15:505-13. 